\title{
Landscape of Future Accelerators at the Energy and Intensity Frontier
}

\author{
M.J. Syphers* and S. Chattopadhyay \\ Northern Illinois University, USA \\ Fermi National Accelerator Laboratory, ${ }^{\dagger} U S A$ \\ E-mail: msyphers@niu.edu, schaterji@niu.edu
}

\begin{abstract}
An overview is provided of the currently envisaged landscape of charged particle accelerators at the energy and intensity frontiers to explore particle physics beyond the standard model via 1-100 TeV-scale lepton and hadron colliders and multi-Megawatt proton accelerators for short- and longbaseline neutrino experiments. The particle beam physics, associated technological challenges and progress to date for these accelerator facilities (LHC, HL-LHC, future $100 \mathrm{TeV}$ p-p colliders, Tev-scale linear and circular electron-positron colliders, high intensity proton accelerator complex PIP-II for DUNE and future upgrade to PIP-III) are outlined. Potential and prospects for advanced "nonlinear dynamic techniques" at the multi-MW level intensity frontier and advanced "plasmawakefield-based techniques" at the TeV-scale energy frontier and are also described.
\end{abstract}

38th International Conference on High Energy Physics

3-10 August 2016

Chicago, USA

\footnotetext{
* Speaker.

$\dagger$ Operated by Fermi Research Alliance, LLC under Contract No. DE-AC02-07CH11359 with the United States Department of Energy.
} 
While accelerator-based high energy physics programs continue to push toward higher particle energies, the demands for greater beam intensity and beam brightness press onward in searches for new physics or in more precise measurements of particle properties. The current landscape of charged particle accelerators at the energy and intensity frontiers is tending toward 1-100 TeV-scale lepton and hadron colliders and multi-Megawatt proton accelerators for short- and long-baseline neutrino experiments. Below we provide a sampling of the associated technological challenges and progress to date for these frontier accelerator facilities, touching upon advanced "nonlinear dynamic techniques" at the multi-MW level intensity frontier, and the potential prospects for advanced "plasma-wakefield-based techniques" at the TeV-scale energy frontier.

\section{Intensity Frontier}

Presently, the high intensity proton/neutrino facilities around the world include the original CNGS facility at CERN with more than $400 \mathrm{~kW}$ of proton beam power; the original Fermilab Main Injector in USA with more than $600 \mathrm{~kW}$ of beam power achieved to date; currently operating JPARC facility in Japan with more than $300 \mathrm{~kW}$ of proton beam power; and an evolved facility at Fermilab known as the PIP (Proton Improvement Plan) which has achieved a proton beam power of more than $700 \mathrm{~kW}$ as of 2016 . The envisioned future evolution of this latest facility includes an upgrade to a $\geq 1.2 \mathrm{MW}$ facility known as PIP-II by about 2025 in support of the initial implementation of the Deep Underground Neutrino Experiment (DUNE) being put together by an international collaboration, with an eventual aspiration of a post-PIP-II upgrade to multi-MW capability [1], [2]. This last aspiration poses significant accelerator technology and beam physics challenges and can only be realized by commensurate research and development of high intensity beams at targeted and specially directed beam test facilities, as planned in the Fermilab Accelerator Science and Technology (FAST) facility, in particular its Integrable Optics Test Accelerator (IOTA) [3], [4].

The current PIP facility provides for full $15 \mathrm{~Hz}$ operation of the $8 \mathrm{GeV}$ Booster injected with a $400 \mathrm{MeV}$ normal conducting linear accelerator, at $4 \times 10^{12}$ protons per pulse with an average power of 0.45 to $0.7 \mathrm{MW}$, for neutrinos, muons, test beams, etc.The near future PIP-II plans are based on a $20 \mathrm{~Hz}$ operation of the $8 \mathrm{GeV}$ Booster injected with a newly installed $800 \mathrm{MeV}$ superconducting linear accelerator, with $6.5 \times 10^{12}$ protons per pulse and an average power reaching up to $1.2 \mathrm{MW}$.

The typical research and development areas of PIP-II development includes: (i) special commissioning of an RFQ-based proton injector with beam energy of $2.087 \mathrm{MeV}$ within a precision of $0.02 \mathrm{MeV}$ with 95\% transmission; (ii) proton injector Medium Energy Beam Transport (MEBT) line; (iii) robust operation of the Booster at $20 \mathrm{~Hz}$ and (iv) successful robust operation of the superconducting $800 \mathrm{MeV}$ linear accelerator.

To enable multi-MW beam power, particle losses must be kept well below $0.1 \%$ at these high intensities (e.g. $<0.06 \%$ for post-PIP-II at $2.5 \mathrm{MW}$ ). This must be compared to the present level of 3-5\% in the current Booster and Main Injector. This is a challenging task indeed. Thus there is need to develop tools for Coulomb self-force "space-charge" compensation and countermeasures, beam "halo" control techniques and techniques to ensure single particle and collective coherent beam stability.

The path beyond PIP-II for a multi-MW proton beam option is exploratory at this stage. One technically easiest approach is to simply replace the whole injector complex with a full energy 
$8 \mathrm{GeV}$ superconducting linear accelerator. This approach, while straight forward technically, is likely to be cost-intensive. Significant developments of cost-saving performance measures, e.g. higher quality-factor linear accelerator cavities, simpler and cheaper superconducting cryomodule design, cheaper cryogenic solutions, etc., must be found before this solution can be adopted. In addition, it is not clear a priori that cheaper room-temperature innovative linear accelerator technologies are not competitive, were we to start from ground zero.

Yet, a fascinating alternative could be within the realm of possibilities, but this will require intense focused $R \& D$ for a period of time before its merits/demerits can be appropriately evaluated. This alternative approach involves developing an innovative Booster concept, dubbed as "i-Booster" by Fermilab accelerator scientists, which will replace the old $8 \mathrm{GeV}$ Booster with a new 8-12 GeV "smart" Rapid Cycling Synchrotron (RCS). This new Booster would depend on a specially designed highly nonlinear circular synchrotron, where the nonlinearities in the motion of a proton in the intense proton beam arising from its self-forces due to Coulomb repulsion are very cleverly compensated by an innovatively designed nonlinear yet "integrable" beam electromagnetic focusing lattice, so eliminating deleterious effects of a multitude of dynamical resonances and resulting diffusion, stochasticity and eventual particle loss from the beam which are the ultimate "beam current" limiting phenomena in present accelerators.

The Integrable Optics test Accelerator (IOTA) within the FAST accelerator complex at Fermilab, shown in Fig. 1 below, is envisaged to provide the very necessary test beam facility to address the challenging $R \& D$ above. The specially designed nonlinear yet "integrable" test synchrotron will be a 40 meter circumference ring able to store high quality $150 \mathrm{MeV}$ electrons injected from the FAST $250 \mathrm{MeV}$ superconducting linear accelerator complex for precise characterization of its nonlinear dynamical transfer map characteristics. Subsequently, the ring can be filled with very intense pulses of $2.5 \mathrm{MeV} / \mathrm{c}$ protons and its subsequent nonlinear dynamics of beam "halo" generation, particle loss, resonance diffusion, streaming and stochasticity can be thoroughly investigated via appropriate diagnostics and control installed in the ring. Three classes of experimental investigation are envisioned in IOTA: (i) tests of integrable optics (with nonlinear magnets in the ring, tested with both electrons and protons and specially designed electron lenses in the ring, tested with only protons); (ii) space-charge compensation schemes (tested with electron lenses and columns); and (iii) characterization of Hamiltonian phase-space diffusion, resonance streaming and ultra-fast stochastic Arnold Diffusion.

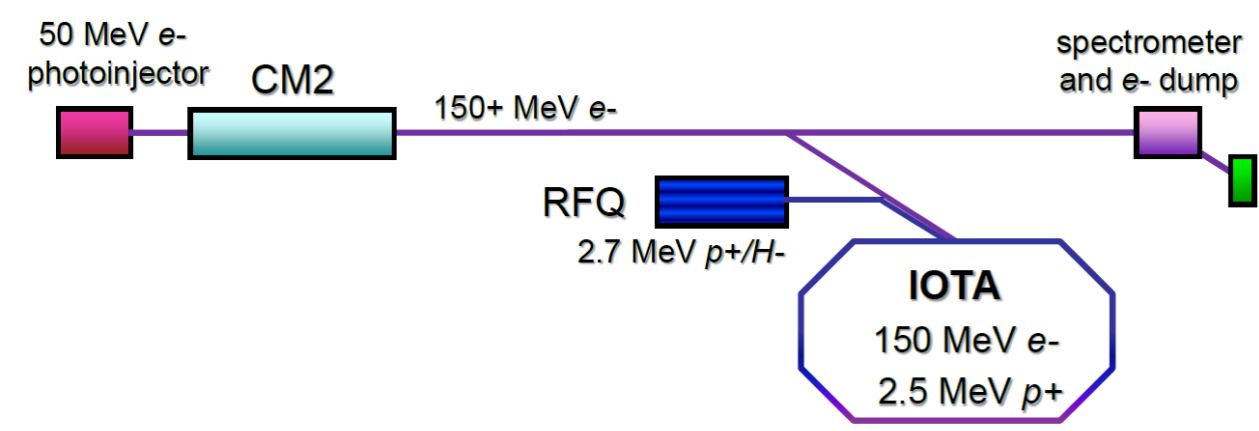

Figure 1: Schematic layout of the Fermilab Accelerator Science and Technology (FAST) facility, including the Integrable Optics Test Accelerator (IOTA). 


\section{Energy Frontier}

The energy frontier is presently dominated by the Large Hadron Collider (LHC) operation at CERN. Having its goals in both energy and luminosity well within reach, the next steps for the LHC involve its high-luminosity upgrade program (HL-LHC) to increase the luminosity by a factor of 10 for the $14 \mathrm{TeV}$ collider. This program has been in the design stage for the past 15 years and component construction has recently been implemented. Physics at the higher luminosities is expected in roughly 2025. With time scales of 20 years required between design and first-physics, a future collider program beyond the HL-LHC dictates that its design begin essentially now.

\subsection{Future Collider Studies}

Designs for linear electron-positron colliders (ILC and CLIC) have become fairly mature over the past decades, with efforts in hardeware development and cost reduction continuing. [5] However, new investigations into future large-scale circular colliders are now going on in the world - the Future Circular Collider Study (FCC) in Europe and the Circular Electron-Positron Collider/Super Proton-proton Collider (CEPC/SPPC) in China. [6] (The FCC study includes hadron collider (hh), lepton collider (ee), and hadron-lepton (he) collider options.) As would be expected, the major hh collider driver would be the development of high-field superconducting magnets, on the scale of 16-20 T, while the ee options succumb to the limits on RF voltage/power and synchrotron radiation.

As an example, the FCC study is looking into a $100 \mathrm{~km}$ circumference ring using $16 \mathrm{~T}$ dipole magnets and achieving an energy of $50 \mathrm{TeV}$ per beam. To achieve its luminosity goal of $10^{34}$ $10^{35} \mathrm{~cm}^{-2} \mathrm{~s}^{-1}$ requires a beam intensity amounting to a total stored beam enery of $8.4 \mathrm{GJ}$ per beam. Energy deposition and collimation will be especially challenging for such a situation, in which even a beam loss of $0.01 \%$ amounts to the total stored energy in a beam of the Tevatron its full energy. Especially challenging for any of these colliders will be a highly-engineered beamradiation vacuum environment, compensation of beam-beam Coulomb effects, controlling beam instabilities and techniques to mitigate effects of synchrotron radiation. These issues are are all at the forefront of research and development world-wide in the context of the FCC studies of 100 $\mathrm{TeV}$ proton-proton and $275 \mathrm{GeV}$ electron-positron colliders under development by the FCC-CERN global collaboration and the $\mathrm{CepC}$ and SppC studies in China along similar lines.

\subsection{Proton-driven Plasma Acceleration: $A W A K E$}

Extensive recent studies and computer simulations have indicated that a high energy proton bunch is capable of exciting strong electromagnetic wakefields in a plasma which can accelerate a bunch of electrons to the energy frontier of up to a $\mathrm{TeV}$ in energy in a single stage of acceleration [8], [9]. If proton beams from existing high energy proton colliders e.g. LHC could be suitably configured to drive an appropriately designed plasma column, one could envision a high energy electron-proton collider with $1 \mathrm{TeV}$ electrons colliding against $7 \mathrm{TeV}$ protons as an energy frontier ep collider to go far beyond conventional QCD investigations [10]. Whether positrons could be accelerated in a similar fashion to enable a $\mathrm{TeV}$-scale electron-positron collider remains an open question due to various positron-specific fundamental beam physics issues, but initial attempts at a preliminary parametric design exists [11]. 
Today's peta-watt lasers or high energy electron beams (e.g. at SLAC) have stored energies of 30-40 Joules/pulse. In contrast a high energy proton beam can store three to four orders of magnitude higher energy per pulse (300 kilo-Joules of energy per proton bunch in the LHC!). For acceleration of $10^{10}$ electrons to $1 \mathrm{TeV}$ via sampling the generated electromagnetic wake field, an energy of a few kilo-Joules only is required. Hence the energy content of existing proton drivers allow us to consider a single stage electron acceleration to $\mathrm{TeV}$ scale energies.

In recognition of the above scaling facts and following many years of serious investigation, a prototype demonstration experiment, AWAKE - using $400 \mathrm{GeV}$ protons from CERN's SPS synchrotron driving a 10 meter plasma column, injected with a well-designed 5- $20 \mathrm{MeV}$ electron beam from a specially designed injector and generating electrons with few GeVs of energy in a few meters of acceleration - has been proposed by the community to CERN and eventually approved [12], [13]. The schematics of this experiment and its location within CERN's SPS synchrotron ring, are shown in Fig. 2. It will be implemented in the SPS beam extraction cave area originally used
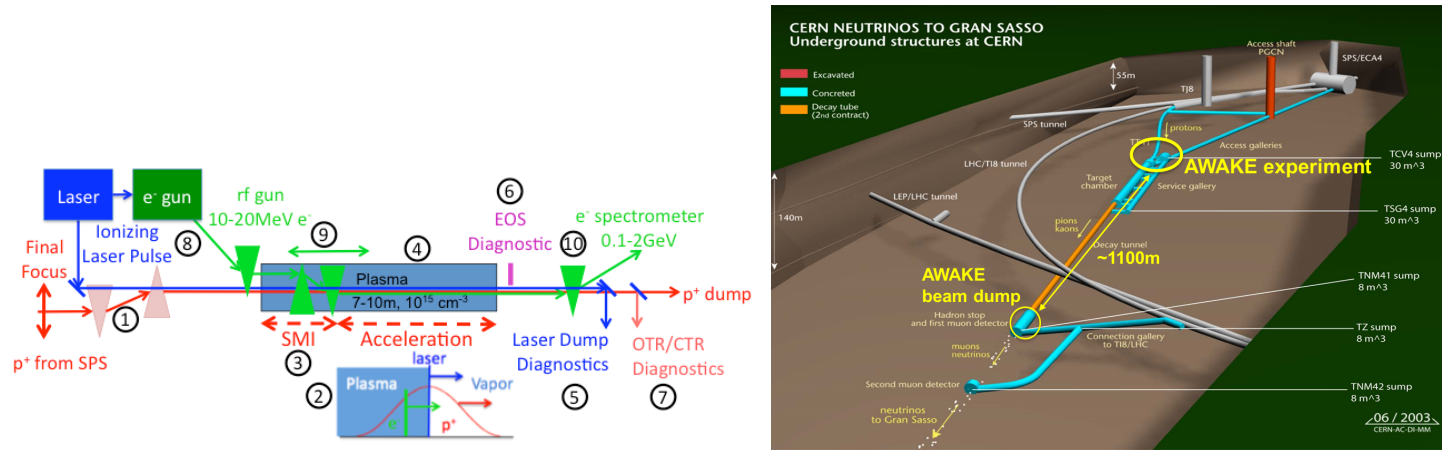

Figure 2: Left: schematics of AWAKE experimental layout with external electron injector, Plasma column, protons injected from SPS and subsequent beam diagnostics; Right: location of AWAKE facility within the SPS original Gran Sasso cave.

for generating neutrinos via a high power target for the Gran Sasso experiment. A pan-European collaboration has been formed for a few years now [9] and commissioning is just beginning. The current AWAKE time line involves initial running in 2016 for 4 weeks equivalent of continuous running with proton-beam commissioning, a Phase 1 of initial data-taking and a Phase 2 of further fabrication and installation of the plasma column and electron injector during calendar years 2017 and 2018, followed by a Long Shutdown for 24 months during calendar years 2019 and 2020, followed by serious systematic wakefield Acceleration studies.

\section{Outlook}

Intensity frontier accelerators are developing in a staged fashion around the world with the goal to provide higher intensity protons, hence neutrinos, for particle physics investigations. In Fermilab in US for example, the accelerator research and development is progressing in phases ranging from PIP, to PIP-II to post-PIP-II with the goal of providing multi-MW proton sources eventually for neutrino physics. In developing the highest intensity neutrino platform via accelerators, we are also enabling access to lower-energy, high intensity sources of muons, kaons, etc., for precision studies of physics beyond the Standard Model (e.g., g-2 measurements of the muon, 
mu-to-e conversion of the muon near a nucleus, CP-violation, flavor-violation, other BSM studies at ever increasing particle rates). The underlying research and development for multi-MW proton beams is taking place on the dual axes of developing more efficient, cost-effective and high performance superconducting linear accelerators on one hand and developing innovative yet technologically conventional synchrotrons that can compensate beam self-Coulomb force effectively on the other.

In the energy frontier, larger synchrotrons utilizing very high-field superconducting magnets (16-20 T), specially engineered beam-radiation vacuum environment, compensation of beam-beam Coulomb effects, controlling beam instabilities and techniques to mitigate effects of synchrotron radiation - are all at the forefront of research and development world-wide in the context of the Future Circular Collider (FCC) studies of $100 \mathrm{TeV}$ proton-proton and $275 \mathrm{GeV}$ electron-positron colliders under development by the FCC-CERN global collaboration and the CepC and SppC studies in China along similar lines.

Finally, proton-driven plasma wakefield accelerators continue to make progress in developing prototype tests of the concept, but an operational energy-frontier particle physics facility is futuristic and speculative at this point in time.

\section{References}

[1] See https://pip2.fnal.gov.

[2] Proton Improvement Plan - II, Fermilab Report Rev 1.1, 12 December (2013).

[3] Focused Workshop on Scientific Opportunities in IOTA, https://indico.fnal.gov/conferenceDisplay.py?confld=9734.

[4] IOTA - a brief parametric profile, Fermilab/FAST Facility document, http://asta.fnal.gov/IOTA/IOTAmeeting/IOTA_Short.pdf.

[5] See http://www.linearcollider.org.

[6] See http://fcc.web.cern.ch, and http://cepc.ihep.ac.cn.

[7] F. Zimmermann, M. Benedikt, D. Schulte, J. Winninger, Challenges for Highest Energy Circular Colliders, proc. IPAC 2014, Conf. C14-06-16, Dresden (2014).

[8] A. Caldwell et al., Nature Physics 5, 363 (2009).

[9] AWAKE Collaboration, A. Caldwell et al., Path to AWAKE: Evolution of a concept, Nucl. Inst. Meth. A, November 2015, arXiv: 1511.09032.

[10] A. Caldwell, M. Wing, VHEeP: a very high energy electron-proton collider, http://link.springer.com/article/10.1140/epjc/s10052-016-4316-1.

[11] G. Xia et al., Collider design issues based on proton-driven plasma wakefield acceleration, Nucl. Inst. Meth. A740, 173-179 (2014).

[12] See http://www.cern.ch/awake.

[13] A. Caldwell et al, CERN-SPSC-2013-013 (2013). 